Artículo

\title{
Análisis bibliométrico del conocimiento científico sobre resiliencia de agroecosistemas
}

\author{
Ismael Quiroz Guerrero ${ }^{1}$ \\ Arturo Pérez Vázquez ${ }^{2 \S}$ \\ Cesáreo Landeros Sánchez ${ }^{2}$ \\ Felipe Gallardo López ${ }^{2}$ \\ Joel Velasco Velasco ${ }^{3}$ \\ Griselda Benítez Badillo ${ }^{4}$
}

${ }^{1}$ Instituto Tecnológico Superior de Zongolica. Carretera a la Compañía s/n km 4, Tepetitlanapa, Zongolica, Veracruz. CP. 95005. (ismael.quiroz.pd254@zongolica.tecnm.mx). ${ }^{2}$ Programa de Postgrado en Agroecosistemas Tropicales-Colegio de Postgraduados. Tepetates, Veracruz, México. CP. 91700. (clandero@colpos.mx; felipegl@colpos.mx). ${ }^{3}$ Colegio de Postgraduados-Campus Córdoba. Veracruz, México. (joel42ts@colpos.mx). ${ }^{4}$ Instituto de Ecología. Xalapa, Veracruz, México. CP. 91070. (griselda.benitez@inecol.mx).

${ }^{\S}$ Autor para correspondencia: parturo@colpos.mx.

\section{Resumen}

Fenómenos globales como el cambio climático amenazan la resiliencia de los agroecosistemas y por tanto la seguridad alimentaria. Por lo anterior, el objetivo del presente trabajo fue analizar el carácter acumulativo y avances de sobresalientes en el conocimiento de la resiliencia de agroecosistemas como base del desarrollo de nuevas tendencias del tema. Se realizó una revisión de literatura con el uso de la base de datos Web of Science considerando criterios como fecha de publicación, disciplinas y revistas científicas. La búsqueda comprende el periodo 1993 al 2020. Se identificaron 188 publicaciones, siendo 2018 y 2019 los años con mayor número de artículos publicados. Las áreas de investigación con más publicaciones en resiliencia son ecología (71), agricultura (52) y ciencias ambientales (44). Respecto a la conectividad basada en el valor de centralidad intermedia, las áreas de mayor interrelación son principalmente: agricultura (0.45), ciencia y tecnología (0.28), ciencias ambientales (0.2) y ecología (0.12). En la dinámica y estudio de la resiliencia sobresale la capacidad de adaptación del agroecosistema. El modelo conceptual de resiliencia analizado facilita su estudio y está integrado por: la precariedad, latitud, resistencia y panarquía. Por tanto, los indicadores para cuantificar la resiliencia en agroecosistemas son heterogéneos y multidimensionales. Se concluye que la resiliencia ha sido estudiada conceptualmente como una propiedad emergente desde el enfoque agroecológico, recientemente desde el enfoque de sistemas socioecológicos en donde se resalta la capacidad adaptativa y a la interdisciplina como medio para resolver los problemas complejos.

Palabras clave: adaptación, regeneración, vulnerabilidad.

Recibido: abril de 2021

Aceptado: mayo de 2021 


\section{Introducción}

Desde la teoría y conceptualmente, los agroecosistemas como unidades de estudio, aparecen en discusiones académicas hasta los años 70's del siglo pasado abordando la interrelación entre los sistemas agrícolas y el ser humano (Harper, 1974; Argueta et al., 2015). Posteriormente, en los años 80's y 90's los estudios sobre agroecosistemas abordaron el diseño de estrategias para el manejo sostenible de los recursos naturales (Conway, 1987) y es a partir del año 2000, que se comenzó a abordar la recurrencia del cambio climático y otras amenazas globales con la aplicación de enfoques integradores de modelaje y planeación, retomando principios pro-ambientalistas como agricultura ecológica, campesina e indígena (Gliessman, 2002; Casanova-Pérez et al., 2015; Mebarki, 2017).

Desde la aparición de la construcción concepto agroecosistema, este ha sido abordado con criterios positivistas-deterministas (Maldonado y Gómez, 2011) y con una perspectiva lineal y simplificada. El enfoque reduccionista de investigación en los agroecosistemas desde su aparición como concepto fue prácticamente contrario a la naturaleza de fenómenos como el cambio climático que ponen en tela de juicio a la predictibilidad.

En esta investigación, el estudio del agroecosistema se abordó como un sistema alopoíetico con una red compleja de interacciones, y con capacidad de adaptación del tipo socioecológico integrado por componentes sociales, económicos, tecnológicos y ambientales en donde la fortaleza de los mismos, el procesamiento de señales e información y la capacidad de adaptación le provee resiliencia expresada como propiedad emergente ante perturbaciones y panarquías del sub y el supra sistema (Mitchell, 2009; Casanova-Pérez et al., 2015; Dekkers, 2015; Gao y Barabási, 2016).

Es común que los agroecosistemas presenten un comportamiento cíclico significativo que ocurre en múltiples escalas (Gunderson, 2008). Para conceptualizar la interacción de escalas cruzadas, las dimensiones del agroecosistema pueden representarse en espacio y tiempo, tal es el caso en el análisis de una región geográfica, un sistema de finca, agroecosistemas con diversos cultivos y ganado, un cultivo o simplemente una especie vegetal o animal (Hart, 1985; Dekkers, 2015).

La teoría de la panarquía puede describir el efecto de indicadores a pequeña escala con su respectiva expresión rápida y de corta duración, y su efecto a mayor escala con una expresión lenta, pero con mayor duración. El efecto de los indicadores puede provenir del micro o macrosistema influyendo en cambios incrementales que ocurren de manera lenta a medida que los sistemas maduran.

Lo anterior conllevará a un cambio adaptativo que ocurre después de las perturbaciones. Sin embargo, existe el riesgo de alcanzar el estado de transformación que podría ocurrir si la resistencia del sistema es rebasada por la perturbación, lo que conllevará a la conducción del agroecosistema a un nuevo estado con tipos de estructuras, procesos y controles diferentes (Gunderson, 2008).

Debido a retos globales para la humanidad como alcanzar la seguridad alimentaria bajo el cambio climático, se considera importante conocer el carácter acumulativo, los avances sobresalientes y la tendencia en el conocimiento de la resiliencia de agroecosistemas como base del desarrollo de nuevas tendencias del tema. 
Por lo que la presente investigación parte de dar respuesta a las siguientes preguntas ¿Cuáles son los dominios, origen de las publicaciones, autores, conceptos, metodologías y teorías para el estudio de resiliencia del agroecosistema? ¿cuál es la prospectiva respecto a la resiliencia de agroecosistemas? y tiene como objetivo determinar el carácter acumulativo y avances sobresalientes en el conocimiento de la resiliencia de agroecosistemas como bases del desarrollo de nuevas tendencias del tema.

Este artículo está dividido en seis partes: 1) estudios sobre resiliencia en agroecosistemas; 2) la conceptualización de la resiliencia en el contexto de los agroecosistemas; 3) los componentes de la resiliencia; 4) los indicadores de resiliencia; 5) los estudios de las etapas de la resiliencia; y 6) la prospectiva del análisis de resiliencia en agroecosistemas.

\section{Materiales y métodos}

Se realizó una revisión bibliográfica utilizando la base de datos Web of Science y criterios de palabras como 'resiliencia' y su asociación con 'agroecosistemas' 'adaptación' 'sistemas agrícolas' y 'agricultura'. Posteriormente, se procedió a depurar los resultados. Una vez identificados los artículos que abordan a la resiliencia como una propiedad emergente de los agroecosistemas, se procedió a revisar e identificar los conceptos y métodos para el análisis de la resiliencia en agroecosistemas. Posteriormente, los artículos fueron cuantificados de acuerdo con el año de publicación.

También, se organizó una base datos en formato txt que incluyó información por autores, tema, país de origen, y citas del autor. Se utilizó la herramienta visual CiteSpace 5.3 con la que se analizaron la frecuencia de citas y se determinó los artículos más conectados mediante co-citas de autores en las referencias de los artículos publicados. De igual manera, el título, resumen, palabras clave fueron analizados para identificar tendencias y conexiones de dominios según los temas de abordaje de la resiliencia.

\section{Resultados}

\section{Estudios sobre resiliencia en agroecosistemas}

En años recientes, el término resiliencia ha tomado importancia y ha sido utilizado en diversas áreas del conocimiento. En el caso de los agroecosistemas y resiliencia (como palabras clave) se han realizado 188 publicaciones desde 1993, siendo 2018 y 2019 los años con más artículos publicados con 30 y 32 artículos respectivamente (Figura 1).

Los artículos sobre resiliencia de agroecosistemas analizados con base a su frecuencia de citas se integraron en 11 dominios que a continuación se describen: ciencias ambientales y ecología (71), agricultura (52), ciencias ambientales (44), ecología (32), ciencia y tecnología (26), agricultura multidisciplinaria (23), ciencia verde y sostenible (20), estudios ambientales (19), biodiversidad y conservación (10), ciencia del suelo (9), agronomía (7), meteorología y ciencias atmosféricas (4) ciencia multidisciplinaria (3) y recursos hídricos, ciencia de plantas, ingeniería ambiental, biología, biomedicina, silvicultura e ingeniería con dos publicaciones respectivamente. 


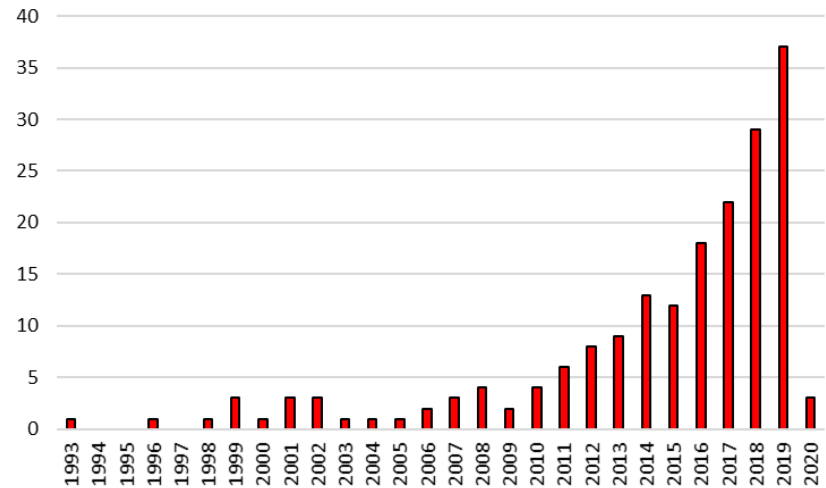

Figura 1. Publicaciones sobre resiliencia de agroecosistemas registradas en la Web of Science de 1993 al 2020.

De acuerdo con la conectividad basada en el valor de centralidad intermedia, las áreas que se relacionan con otras en mayor frecuencia fueron principalmente ciencias ambientales y ecología (0.37), agricultura (0.32), estudios ambientales (0.29) ciencia y tecnología y otros temas $(0.27)$ ecología (0.2) y ciencia ambiental (0.14) (Figura 2). La relación marcada entre las diversas áreas presenta interacciones y realimentaciones entre los problemas sociales, económicos, políticos y ecológicos diferentes escalas espacio-temporales (Castillo y Velázquez, 2015).

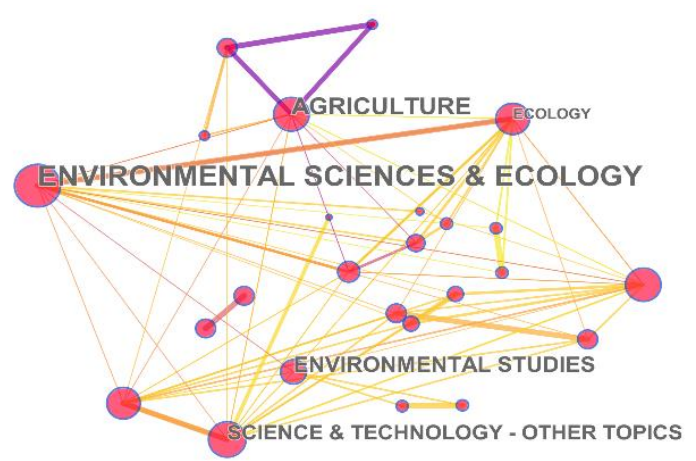

Figura 2. Principales disciplinas que abordan la resiliencia en agroecosistemas de acuerdo con la herramienta CiteSpace.

Los estudios han demostrado que los nodos con altos valores de centralidad de intermediación tienden a identificar potenciales de expansión de límites que pueden conducir a descubrimientos transformadores.

El país de origen de los co-autores que han publicado sobre resiliencia son principalmente de: Estados Unidos de América (34), Francia (20), Australia (10), Canadá (7) y Alemania (6). Siendo Francia y Estados Unidos de América los nodos que conectan e influyen en dos o más grandes grupos (Figura 3) esto se indica mediante el tamaño del cada nodo (Chaomei, 2014). Los resultados mostrados en las redes presentan similitud en los dominios analizados por Hosseini et al. (2016), quienes, al realizar una revisión de literatura sobre resiliencia de sistemas, determinaron la presencia de Psicología como área principal y seguida por las ciencias ambientales y la ecología como áreas desde donde también se ha abordado este concepto. 


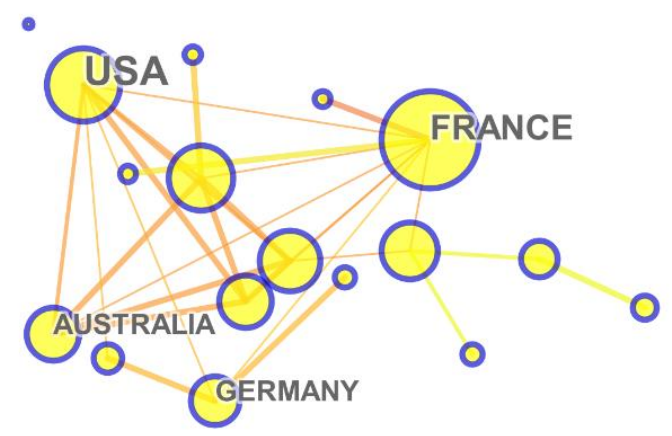

Figura 3. Principales países de origen y valor de centralidad intermedia de co-autores relacionados a las publicaciones de resiliencia de agroecosistemas en el periodo 1993-2020.

Con relación a los autores citados con mayor frecuencia de los 188 artículos, sobresalen Altieri (53), Folke (31), Walker (30), Tilman (29), Holling (26), Gliessman (22), Gunderson (16), Kremen (15), Lal (15), Tscharntke (14), FAO (14), Scheffer (14), Lin (12), Carpenter (12), Adger (11), Van Der (11) y Perfecto (11). Con relación a la centralidad basada en la conectividad de los autores mediante co-citas sobresalen: Altieri (0.24), Hooper (0.2), Walker (0.21), Holling (0.12), Folke (0.11), Swift (0.11), Tilman (0.1), Tscharntke (0.08), Lin (0.06) y Koohafkan (0.06). Posteriormente, se presentan otroscon menor grado de centralidad y estos son: Gliessman (0.04), Gunderson (0.04), Scheffer (0.03), FAO (0.03), Lal (0.02), Carpenter (0.02) y Kremen (0.01).

La intensidad de relación de los autores sobresalientes y aquellos con menor grado de centralidad es por la cantidad de artículos citados que tienen a estos autores en sus referencias (Figura 4). En un contexto más amplio sobre resiliencia de sistemas Hosseini et al. (2016) caracterizó la presencia de autores que estudian la resiliencia desde enfoques cualitativos, semicuantitativos y cuantitativos.

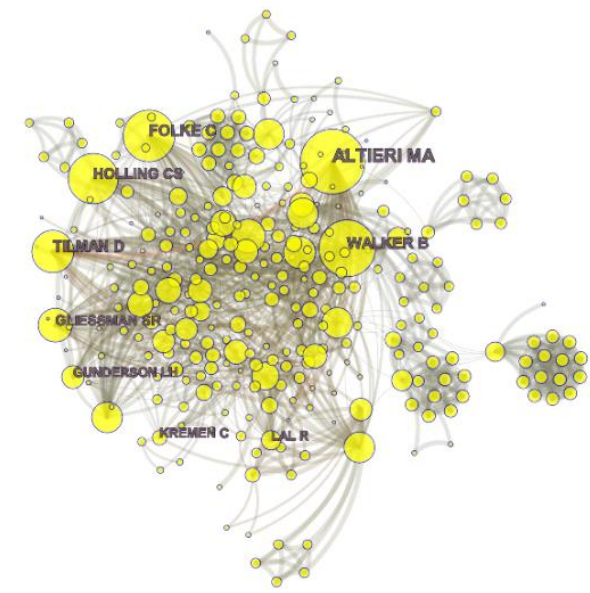

Figura 4. Red que muestra a los principales autores citados en los 188 artículos analizados.

Del total de artículos (188) para el periodo 1993-2020, y de acuerdo con Web of Science, los artículos más citados en el extenso de su base de datos y sin delimitar su relación a agroecosistemas, estos están relacionados con el estudio de la resiliencia del agroecosistema desde el enfoque ecológico (Cuadro 1). 
Cuadro 1. Principales artículos sobre resiliencia de agroecosistemas citados.

\begin{tabular}{lcc}
\hline \multicolumn{1}{c}{ Título de la publicación } & Autores & Total de citas \\
\hline $\begin{array}{l}\text { Perspectivas del paisaje sobre la intensificación agrícola y } \\
\text { la biodiversidad: gestión de servicios de los ecosistemas }\end{array}$ & Tscharntke et al. (2005) & 1915 \\
$\begin{array}{l}\text { Comprender las relaciones entre múltiples servicios } \\
\text { ecosistémicos }\end{array}$ & Bennett et al. (2009) & 846 \\
\hline
\end{tabular}

\section{Conceptualización de resiliencia en el contexto de los agroecosistemas}

La palabra resiliencia proviene del latín resillire que significa saltar hacia atrás y fue utilizada inicialmente en el área de la física para describir la estabilidad de materiales y su resistencia a impactos externos (Holling, 1973; Reid y Boterill, 2013). En la década de los 60's con el crecimiento del pensamiento sistémico el concepto fue utilizado en ecología (Reid y Boterill, 2013) y su definición destaca dos aspectos; el primero, sobre la recuperación y regreso al estado inicial del ecosistema después de un impacto y el segundo, sobre como un sistema ha sido perturbado y se mantiene sin cambiar su función (Bodin y Wilman, 2004; Masterson et al., 2014).

Posteriormente, el concepto fue incorporado a los agroecosistemas y a finales de los 80's y década de los 90's, fue aplicado dentro del marco teórico ecológico, particularmente tomando las bases del concepto de Holling (1973). Retomando el análisis de redes de la (Figura 4) y con base a definiciones de los autores con mayor centralidad se identificaron dos tipos de enfoques para el estudio de la resiliencia: un enfoque agroecológico y otro enfoque de sistemas socioecológicos.

Desde la agroecología y con el objetivo de desarrollar agroecosistemas sostenibles, con menor impacto ambiental, altamente productivos y económicamente viables, se hizo énfasis en la resiliencia como propiedad emergente del agroecosistema basándose en principios como productividad, estabilidad, sustentabilidad, equidad y principios ecológicos (Conway, 1987; Thomas y Kevan, 1993; Gallopín, 1995).

Aunado a lo anterior, la resiliencia fue conceptualizada como un atributo de la salud del agroecosistema y también, fue estudiada desde la relación con las prácticas sustentables, los servicios ambientales y la biodiversidad (Okey, 1996; Altieri, 1999; Tscharntke et al., 2005). El autor Walker (2004) integró al estudio de la resiliencia el concepto de sistemas socio-ecológicos, que extrapolado a los agroecosistemas integra las propiedades emergentes del productor en su interacción con elementos de los sistemas adyacentes.

El concepto de sistemas socio-ecológicos resalta a la adaptabilidad cíclica como la capacidad de los elementos del agroecosistema de influir en la resiliencia mediante la retroalimentación constante que conlleva a construir estructuras colectivas (basadas en la retroalimentación) que aumentan su resistencia y probabilidad de recuperación ante perturbaciones, generando así más información que se incorpora a los elementos del sistema (Davoudi, 2012; Titonell, 2013).

Sin embargo, también se toma en cuenta al macro y microsistema debido a que en ellos pueden existir variables conductoras a eventos de perturbación drástica que podrían rebasar la resistencia del agroecosistema evitando la emergencia de la resiliencia y en vez de eso, se presente un escenario de transformación (Walker, 2004; Folke, 2006; Gunderson, 2008; Folke et al., 2010; Folke, 2016). 
La extensión de nociones de resiliencia en la sociedad tiene límites importantes, particularmente su conceptualización del cambio social. Lo anterior, se debe a la falta de atención de problemas normativos y epistemológicos que subyacen a la noción de resiliencia social.

Por lo que el papel del conocimiento en las intersecciones entre las dinámicas ambientales y sociales ayuda a abordar las cuestiones normativas y sus respectivos valores como parte integral del desarrollo y funcionamiento del sistema socioecológico. Por lo anterior, resulta necesario desarrollar métodos de sociología que permitan robustecer el estudio de seres humanos con relación a su comportamiento bajo escenarios de estrés ocasionados por fenómenos externos (Cóte, 2011).

Es probable que los métodos unificados para el estudio de la resiliencia en los agroecosistemas aún no están definidos. Lo anterior coincide con una serie de definiciones sobre resiliencia de agroecosistemas y con propuestas de variables con los cuales se podría abordar este fenómeno.

\section{Componentes de la resiliencia}

La definición de resiliencia resulta confusa debido a las connotaciones disciplinarias en las que ha sido utilizada. Desde un enfoque de sistemas socioecológicos, la resiliencia es un componente de la trayectoria de los agroecosistemas (Figura 5) y se operacionaliza con cuatro componentes propuestos por Walker et al. (2004): latitud. Se define como la cantidad máxima que se puede cambiar al sistema antes de perder su capacidad de recuperación. Básicamente, es el ancho de la cuenca de atracción. Una cuenca ancha significa que el sistema puede experimentar un mayor número de estados sin alcanzar la transformación, resistencia. Es la facilidad o dificultad para cambiar el sistema.

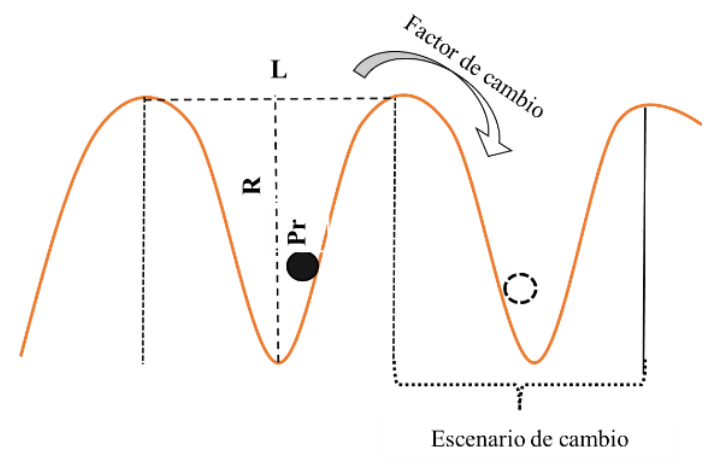

Figura 5. Límites de un sistema socioecológico para expresar resiliencia, donde: $L=$ longitud, $R=$ resistencia y $\operatorname{Pr}=$ precariedad (Walker et al., 2004).

Está relacionado con la topología de la cuenca. Las cuencas profundas de atracción indican que se requieren mayores fuerzas o perturbaciones para cambiar el estado actual a otro, precariedad. Es la trayectoria actual del sistema, la cual lo ubica respecto a un límite o 'umbral' que, si es rebasado, dificulta o imposibilita la recuperación (Pr), panarquía. Proceso de influencia sobre el sistema causado por los estados y la dinámica de los (sub) sistemas en escalas por encimay por debajo de la escala de interés (Pa), (Walker et al., 2004). 
En el contexto comunitario, Masterson et al. (2014) propone tres dimensiones dentro del proceso de resiliencia: 1) la resistencia o robustez, es la habilidad del sistema capital de absorber, cambiar o resistir los impactos por fenómenos naturales, lo que implica una reducción de probabilidades de fallas del sistema; 2) dimensión denominada rapidez, se refiere a la trayectoria de recuperación y a la rapidez con la que el sistema recupera su estado previo al impacto; y 3) se refiere al incremento de la capacidad de resiliencia mediante el aprendizaje o la capacidad de adaptación.

Twigg (2007) propone diversas dimensiones o componentes para sistemas sociales (gobernabilidad, evaluación del riesgo, conocimiento y educación, gestión de riesgos y disminución de la vulnerabilidad, preparación y respuesta para desastres) que una comunidad debe reunir para aumentar su capacidad de resiliencia, dando prioridad a estrategias que permitan a los sistemas actuar por sí mismos y fortalecer sus capacidades, antes que concentrarse en su vulnerabilidad ante el desastre o sus necesidades en una emergencia.

Desde una perspectiva social, la sostenibilidad y la resiliencia de una comunidad, depende de su capacidad para accesar y utilizar de la mejor forma su capital económico, político y civil ante a una perturbación (Akter y Mallick, 2013). Otros factores importantes de las dimensiones de la resiliencia son: autonomía, seguridad alimentaria, niveles de nutrición adecuados, salud y educación por mencionar algunos, además de la versatilidad de modificar la estructura de su sistema según se requiera ante escenarios de desastre (De La Fuente, 2016).

\section{Indicadores de resiliencia}

Todo estudio de resiliencia, sin lugar a duda debe de partir de las preguntas ¿qué hace resiliente a un agroecosistema?, ¿cuáles son esos atributos del sistema que le confieren mayor o menor resiliencia? y ¿qué indicadores son intrínsecos y extrínsecos al agroecosistema? Sin embargo, es probable que exista confusión sobre qué aspecto de la resiliencia se está midiendo y qué tipo de indicadores se están considerando debido a que la resiliencia puede presentarse en un periodo de tiempo posterior o en una escala particular a expensas de la capacidad de recuperación del sistema.

Para evitar dicha confusión es importante tener claro la escala y el periodo de tiempo en el que se aborda el estudio de la resiliencia (Carpenter et al., 2001; Hernández et al., 2002). Para facilitar el análisis, los indicadores sobresalientes presentados en diferentes investigaciones fueron agrupados en siete categorías: sistemas socioecológicos (Cabell y Oelofse, 2012), agroecológicos (Bruneau et al., 2003; Altieri y Nicholls, 2012; Altieri y Nnicholls, 2015), sociales (Rose, 2007; Rose, 2009; Cabell y Oelofse, 2012; CENAPRED, 2015; Hosseini et al., 2016), capacidades comunitarias (Rose, 2007; Masterson et al., 2014; CENAPRED, 2015; Qasim, 2016), económicas (Rose, 2007; Rose, 2009), institucionales (Rose, 2007; CENAPRED, 2015; Qasim et al., 2016) y de infraestructura (Rose, 2007; CENAPRED, 2015; Qasim et al., 2016).

Cada categoría se relaciona al agroecosistema directa o indirectamente y su influencia determina la respuesta del agroecosistema ante una crisis. Finalmente, una vez seleccionados los indicadores de resiliencia es importante estandarizar los valores obtenidos para lo que se puede hacer uso de la ecuación (1) propuesta por Hahn et al. (2009): $I_{V}=\frac{I_{a}-I_{\min }}{I_{\max }-I_{\min }}$ 1) en donde $I_{V}$ es el valor estandarizado del indicador, $\mathrm{I}_{\mathrm{a}}$ el valor para un agroecosistema en particular, $\mathrm{I}_{\min }$ para el valor mínimo del total de agroecosistemas, $\mathrm{I}_{\max }$ para el valor máximo del total de los agroecosistemas (Hahn et al., 2009). 
En términos de prevención de desastres, es sustancial enfocarse en aumentar la robustez del agroecosistema y disminuir la vulnerabilidad debido a que estas variables son inversamente proporcionales a la resiliencia. Probablemente, un agroecosistema con baja vulnerabilidad podría no sufrir daño alguno o un daño mínimo.

En el estudio y diseño de los agroecosistemas resilientes, es importante abordar los procesos alopoíeticos para conocer como es la dinámica de interacción con el ambiente o los sistemas externos como promotores de cambios de estado del sistema o de auto-organización interna.

En la etapa de adaptación (previo a la expresión de resiliencia), el agroecosistema modifica o cambia algunos elementos de su estructura en respuesta al ambiente que le rodea, por lo que cada vez que esta fase termina y un agroecosistema es resiliente, puede significar tres cosas: 1) que el agroecosistema presenta una auto-organización interna; 2) que la resiliencia es un comportamiento emergente; y 3) que es un cambio de estado resultado de un agroecosistema con alta entropía (Dekkers, 2015). Finalmente, se debe fomentar a la interdisciplina como instrumento metodológico para abordar la resiliencia.

Esta representa un marco conceptual y metodológico común, derivado de una concepción compartida de la relación ciencia-sociedad, que permitirá definir la problemática a estudiar bajo un mismo enfoque, sin dejar de lado la especialización de cada uno de los miembros del equipo de investigación (García, 2006).

\section{Conclusiones}

El estudio de la resiliencia como propiedad emergente en los agroecosistemas se ha incrementado significativamente en los últimos diez años. Los principales dominios en los que se agrupan las publicaciones son: ciencias ambientales, ecología y agronomía. Los autores de publicaciones como Altieri, Walker y Folke son quienes presentaron mayor cantidad de co-citas por lo que se pueden considerar como los principales autores con influencia en el tema. Actualmente, la socioecología ha integrado los fundamentos de la agroecología y las disciplinas sociales y económicas para estudiar la resiliencia.

El reto actual, es analizar la resiliencia del agroecosistema reforzando los estudios sobre los elementos sociales cualitativos con relación a la capacidad de adaptación y el aprendizaje en el contexto de espacio y tiempo. La definición de resiliencia ha evolucionado y continuará aumentando en complejidad a medida que se le incorporen más elementos. También, es necesario unificar un método para todas las dimensiones relacionadas a la resiliencia del agroecosistema. La teoría de sistemas facilita el estudio de la resiliencia del agroecosistema y la herramienta conceptual más sobresaliente para su estudio es la interdisciplina debido a que considera a los elementos de un sistema como inter-definidos y en concepción común sobre la problemática general a estudiar.

Entre más vulnerable sea un agroecosistema el daño en su estructura será mayor y por consiguiente la recuperación requerirá más tiempo, pero también si la perturbación rebasa el umbral de resistencia del agroecosistema este se transformará. 


\section{Agradecimientos}

Esta obra fue publicada gracias al apoyo del Consejo Veracruzano de Investigación Científica y Desarrollo Tecnológico.

\section{Literatura citada}

Altieri, M. 1999. The ecological role of biodiversity in agroecosystems. Agric. Ecosystems Environ. 74(1):19-31. https://doi.org/10.1016/S0167-8809(99)00028-6.

Altieri, M. A. and Nicholls, C. 2012. The scaling up of agroecology: spreading the hope for food sovereignty and resiliency A contribution to discussions at Rio+20 on issues at the interface of hunger, agriculture, environment and social prepared by the scaling up of agroecology: spread. Socla. $20 \mathrm{p}$.

Altieri, M. A.; Nicholls, C.; Henao, I. A. and Lana, M. A. 2015. Agroecology and the design of climate change-resilient farming systems. Agron. Sustainability Developtment. 35(3):869-890. https://doi.org/:10.1007/s13593-015-0285-2.

Argueta, Q.; Orozco-Ramírez Q.; González, S. M. V.; Morales, H. J.; Gerritsen, P. R.W.; Escalona, M.; Rosado-May, F. J.; Sánchez-E, J.; Martínez, T. S. S.; Sánchez-Sánchez, C. D.; Arzuffi, B. R.; Castrejón, A. F.; Morales, H.; Soto, P. L.; Mariaca, M. R.; Ferguson, B.; Rosset, P.; Ramírez, T. H. M.; Jarquín, G. R.; Moya, G. F.; González-Esquivel, C. and Ambrosio, M. 2015. Historia de la agroecología en México. Agroecología. 10(2):9-17.

Bodin, P. and Wiman, B. L. 2004. Resilience and other stability concepts in ecology: notes on their origin, validity and usefulness. ESS Bulletin. 2(2):33-43.

Bruneau, M.; Chang, S. E.; Lee, T. R.; O’Rourke, G. C.; Reinhorn, T. D.; Shinozuka, A. M; Tierney, M. K.; Wallace, W. A. and Von-Winterfeldt, D. 2003. A framework to quantitatively assess and enhance the seismic resilience of communities. Earthquake Spectra. 19(4):733-752. https://doi.org/10.1193/1.1623497.

Cabell, J. F. and Oelofse, M. 2012. An indicator framework for assessing agroecosystems resilience. Ecol. Soc. 17(1):18. http://dx.doi.org/10.5751/ES-04666-170118.

Carpenter, S.; Walker, B.; Anderies, J. M. and Abel, N. 2001. From metaphor to measurement: resilience of what to what? Ecosystems. 4(8):765-781. https://doi.org/ 10.1007/s10021001-0045-9.

Casanova-Pérez, L.; Martínez-Dávila, J. P.; López-Ortiz, S.; Landeros-Sánchez, C.; LópezRomero, G. y Peña-Olvera, B. 2015. El agroecosistema comprendido desde la teoría de sistemas sociales autopoiéticos. Rev. Mex. Cienc. Agríc. 6(4):855-865. https://doi.org/ 10.29312/remexca.v6i4.625.

Castillo, V. L. y Velázquez D. T. 2015. Sistemas complejos adaptativos, sistemas socioecológicos y resiliencia. Quivera. 17(2):11-32.

Chaomei, C. 2014. The CiteSpace manual. College of computing and informatics. Drexel University. Philadelphia, United States of America. 84 p. http://cluster.ischool.drexel.edu/ $\sim$ cchen/citespace/CiteSpaceManual.pdf.

Conway, G. R. 1987. The properties of agroecosystems. Agric. Systems. 24(2):95-117.

Cóte, M. and Nightingale, A. J. 2011. Resilience thinking meets social theory: situating social change in social-ecological systems research. Progress in Human Geography. 36(4):475-489. https://doi.org/10.1177/0309132511425708. 
Davoudi, S.; Shaw, K.; Haider, L. J.; Quinlan, A. E.; Peterson, G. D.; Wilkinson, C.; Fünfgeld, H.; McEvoy, D.; Porter, L. and Davoudi, S. 2012. Resilience: a bridging concept or a Dead End. In: Davoudi S. and Porter, L. Reframing' resilience: challenges for planning theory and practice interacting traps: resilience assessment of a pasture management system in Northern Afghanistan urban resilience: what does it mean in planning practice. Planning Theory Practice. 13(2):299-333. https://doi.org/10.1080/14649357.2012.677124.

Dekkers, R. 2015. Applied systems theory. Switzerland: Springer International. 244 p.

De la Fuente Olguín, J. 2016. ¿Cómo se construye un indicador de desempeño? Boletín del Instituto de Ingeniería Industrial. Santiago, Chile. 27 p.

Folke, C. 2006. Resilience: the emergence of a perspective for social-ecological systems analyses. Global Environ. Change. 16(3):253-267. https://doi.org/10.1016/j.gloenvcha.2006.04.002.

Folke, C.; Carpenter, S. R.; Walker, B.; Scheffer, M.; Chapin, T. and Rockstrom, J. 2010. Resilience thinking: integrating resilience, adaptability and transformability. Ecol. Soc. 15(4):20. http://www.ecologyandsociety.org/vol15/iss4/art20/.

Folke, C. 2016. Resilience. Ecology \& Society 21(4):44. https://doi.org/10.5751/ES-09088210444.

Gallopín, G. C. 1995. Agroecosystem health: A guiding concept for agricultural research? In: Nielsen, N. O. (Ed.). Agroecosystem health: Proceedings of an International Workshop Guelph, Ontario. University of Guelph. 51-65 pp.

Gao, J.; Barzel, B. and Barabási, A. L. 2016. Universal resilience patterns in complex networks. Nature. 530(1):307-312. https://doi.org/10.1038/nature16948.

García, R. 2006. Sistemas complejos conceptos, métodos y fundamentación epistemológica de la investigación interdisciplinaria. Primera edición, Barcelona, España, 201 p.

Gliessman-Stephen, R. 2002. Agroecología: procesos ecológicos en agricultura sostenible. Turrialba, Costa Rica: Sleeping Bear Press. 380 p.

Gunderson, L. 2008. Panarchy. Encyclopedia of ecology 1(2):612-616.

Hahn, M. B.; Riederer, A. M. and Foster, S. O. 2009. The Livelihood vulnerability index: A pragmatic approach to assessing risks from climate variability and change -a case study in Mozambique. Global Environ. Change. 19(1):74-88. https://doi.org/10.1016/j.gloen vcha.2008.11.002.

Harper, J. L. 1974. Agricultural ecosystems. Agro-Ecosystems. 1(1):1-6.

Hart, R. D. 1985. Conceptos básicos sobre agroecosistemas. Centro Agronómico Tropical de Investigación y de Enseñanza (CATIE). Turrialba, Costa Rica. 160 p.

Hernández, A. J. Urcelai, A. y Pastor, J. 2002. Evaluación de la resiliencia en ecosistemas terrestres degradados encaminada a la restauración ecológica. II Reunión Española de Ciencias en Sistemas. Madrid, España. 1-10 p.

Holling, C. S. 1973. Resilience and stability of ecological systems. annual reviews of ecology and systematic. 4(1):1-23. https://doi.org/10.1146/annurev.es.04.110173.000245.

Hosseini, H., Barker, K. and Ramirez-Marquez, J. E. 2016. A review of definitions and measures of system resilience. Reliability Eng. Systems Safety. 145(1):47-61. https://doi.org/ 10.1016/j.ress.2015.08.006.

Maldonado, C. E. y Gómez, N. 2011. El mundo de las ciencias de la complejidad: una investigación sobre qué son, su desarrollo y sus posibilidades. Universidad del Rosario. Bogotá Colombia. 1-134 pp.

Masterson, J. H.; Peacock, W. G.; Zandt, S. S. V.; Grover, H.; Schwarz, L. F. and Cooper, J. T. C. 2014. Planning for community resilience: a handbook for reducing vulnerability to disasters. Washington, DC: Island Press. 1-58 pp. 
Mebarki, A. 2017. Resilience: Theory and metrics -a metal structure as demonstrator. Engineering Structures. 138(9):425-433. https://doi.org/10.1016/j.engstruct.2017.02.026.

Mitchell, M. 2009. Complexity: a guide tour. New York: Oxford University Press. 1-94 pp.

Okey, B. 1996. System approaches and properties, and agroecosystem health. J. Environ. Management. 48(2):187-199.

Qasim, S. M.; Shrestha, R. P.; Khan, A. N.; Tun, K. and Ashraf, M. 2016. Community resilience to flood hazards in Khyber Pukhthunkhwa province of Pakistan. Inter, J. Disaster Risk Reduction. 18(1):100-106. https://doi.org/10.1016/j.ijdrr.2016.03.009.

Reid, R. and Botterill, L. C. 2013. The multiple meanings of resilience: an overview of the literature. Australian J. Public Administration. 72(1):31-40. https://doi.org/10.1111/14678500.12009.

Thomas, V. G. and Kevan, P. G. 1993. Basic principles of agroecology and sustainable agriculture. J. Agric. Environ. Ethics. 6(1):1-19.

Titonell, P. 2013. Livelihood strategies, resilience and transformability in African ecosystems. Agric. Systems 126(1):3-14. https://doi.org/10.1016/j.agsy.2013.10.010.

Tscharntke, T.; Klein, A. M.; Kruess, A.; Dewenter, I. S. and Thies, C. 2005. Landscapes perspectives on agricultural intensification and biodiversity-ecosystems service management. Ecology Letters. 8(8):857-874. https://doi.org/10.1111/j.14610248.2005.00782.x.

Twigg, J. 2007. Características de una comunidad resiliente ante los desastres. Primera edición. Departamento para el desarrollo Internacional del Gobierno del Reino Unido. Londres Inglaterra. $42 \mathrm{p}$. 